\title{
Focal Segmental Glomerulosclerosis with a Mutation in the Mitochondrially Encoded NADH Dehydrogenase 5 Gene: A Case Report
}

\section{Tsukasa Naganuma}

Yamanashi Prefecture Central Hospital: Yamanashi Kenritsu Chuo Byoin

Toshiyuki Imasawa ( $\nabla$ imasawa@nifty.com )

National Hospital Organization Chiba-Higashi National Hospital https://orcid.org/0000-0002-6620-7069

Ikuo Nukui

Yamanashi Prefecture Central Hospital: Yamanashi Kenritsu Chuo Byoin

Masakiyo Wakasugi

Yamanashi Prefecture Central Hospital: Yamanashi Kenritsu Chuo Byoin

Hiroshi Kitamura

National Hospital Organization Chiba-Hogashi National Hospital

Yukiko Yatsuka

Juntendo University: Juntendo Daigaku

Yoshihito Kishita

Juntendo University: Juntendo Daigaku

Yasushi Okazaki

Juntendo University: Juntendo Daigaku

Kei Murayama

Chiba Children's Hospital

Yoshimi Jinguji

Yamanashi Prefecture Central Hospital: Yamanashi Kenritsu Chuo Byoin

\section{Case Report}

Keywords: focal segmental glomerulosclerosis, mitochondrial nephropathy, NADH dehydrogenase 5, Complex I, case report

Posted Date: November 24th, 2020

DOI: https://doi.org/10.21203/rs.3.rs-112927/v1

License: (9) This work is licensed under a Creative Commons Attribution 4.0 International License. Read Full License 


\section{Abstract}

Background: NADH dehydrogenase 5 (ND5) is one of 44 subunits forming Complex I in the mitochondrial respiratory chain. Therefore, a mitochondrially encoded ND5 (MT-ND5) gene mutation causes mitochondrial oxidative phosphorylation (OXPHOS) disorder, resulting in the development of mitochondrial diseases, such as mitochondrial encephalomyopathy, lactic acidosis, and stroke-like episodes. Although focal segmental glomerulosclerosis (FSGS) is induced by several mitochondrial diseases, no reports have yet confirmed that an MT-ND5 mutation causes FSGS.

Case presentation: A Japanese woman at 29 years old was found to have proteinuria and renal dysfunction in an annual health check-up for the first time. Because her proteinuria and renal dysfunction were persistent, she was admitted for a kidney biopsy to investigate the reason at 33 years of age. Although there were no abnormal neurological findings, she was diagnosed with sensorineural hearing loss by an otolaryngologist. Renal histology showed perihilar variant-type FSGS with podocytes filled with abnormal mitochondria. In addition, the renal pathological findings showed granular swollen epithelial cells (GSECs) in tubular cells, age-inappropriately disarranged and irregularly sized vascular smooth muscle cells (AiDIVs), and red-colored podocytes (ReCPos) by acidic dye, which are assumed to be characteristic changes of mitochondrial nephropathy. Genetic analysis using peripheral mononuclear blood cells and urine sediment cells detected the m.13513 G>A variant, which has already been confirmed as a pathogenic variant of the MT-ND5 gene. The heteroplasmy rates of the blood cells and urine sediments were $10.3 \%$ and $62.2 \%$, respectively. Therefore, this patient was diagnosed with FSGS due to an MTND5 mutation.

Conclusion: This is the first case report to show that a gene mutation encoding subunits of Complex I causes FSGS with podocytes filled with abnormal mitochondria. The routine evaluation of GSECs, AiDIVs, and ReCPos is expected to be useful for detecting mitochondrial nephropathies, which could be latent in etiology-unknown FSGS or glomerulosclerosis cases.

\section{Background}

Mitochondrial diseases/disorders are rare diseases thought to occur in every 1 in 5000 births (1). The main role of mitochondria is the biosynthesis of adenosine triphosphate (ATP), the main energy source of cells, through oxidative phosphorylation (OXPHOS) using the mitochondrial respiratory chain (MRC) complex. Therefore, mitochondrial disorders are nearly synonymous with mitochondrial respiratory chain disorders (MRCDs) (2). As the genes related to the MRC complex are encoded not only in mitochondrial DNA (mtDNA) but also in nuclear DNA (nDNA), mitochondrial diseases can occur in cases of mtDNA mutations or nDNA mutations. Indeed, approximately $25 \%$ of mitochondrial disorders are due to mtDNA mutations, whereas the rest are due to mutations in $\operatorname{nDNA}(3,4)$.

Because OXPHOS is absolutely mandatory for all cells that require energy, the clinical phenotypes of mitochondrial diseases are versatile, presenting as encephalopathy $(5,6)$, myopathy (7), cardiomyopathy (8), hepatopathy (9), deafness (10), and diabetes mellitus (11). In addition, mitochondrial nephropathy, which occurs owing to an MRCD of the nephron, has also been reported $(12,13)$. Reports on mitochondrial nephropathy due to mtDNA mutations related to mitochondrial tRNA, such as m.3243A > G, were abundant before 2005. However, technological innovations in genetic analysis have facilitated the analysis of new gene mutations that cause mitochondrial nephropathy. Indeed, since 2006, reports have been published concerning mitochondrial nephropathy due to nDNA mutations related to CoQ10 synthesis, such as COQ2 (14, 15), PDSS2 (16), COQ6 (17), and COQ8b (18). Recently, two mtDNA mutations that encode subunit proteins of MRC complexes have been reported to cause mitochondrial nephropathy. One is the mitochondrially encoded ATP synthase membrane subunit 6 (MTATP6) gene (19). The other is the mitochondrially encoded cytochrome c oxidase I (MT-CO1) gene, that encodes the main subunit protein of the cytochrome c oxidase complex, Complex IV (20). Furthermore, an mtDNA mutation of mitochondrially encoded NADH dehydrogenase 5(MT-ND5), that encodes one of 44 subunits composing Complex I and the largest enzyme of the MRC, was also recently reported as a cause of mitochondrial nephropathy (21). In the report, the main pathological findings were tubulo-interstitial nephropathy. Although the authors of the report assumed that one of three cases was 
accompanied with "secondary" focal segmental glomerulosclerosis (FSGS), neither apparent glomerular lesions nor podocytes with abnormal mitochondria were detected.

Here, we report the first case of mitochondrial nephropathy with apparent glomerular lesions due to an MT-ND5 gene mutation. The renal pathology of this case showed FSGS with increased abnormal mitochondria in the glomerular podocytes and glomerular parietal epithelial cells of the Bowman's capsule. In this report, we propose characteristic pathological findings which should become clues suggesting mitochondrial nephropathy.

\section{Case Presentation}

This case occurred in a Japanese woman, who had been born at 39 weeks of gestation with a birthweight of $3210 \mathrm{~g}$. She had no remarkable medical history until 29 years of age, at which point, an annual health checkup revealed proteinuria (1+) for the first time by a urine dipstick and a high serum creatinine $(\mathrm{sCr})(1.01 \mathrm{mg} / \mathrm{dL})$ level. Her estimated glomerular filtration rate (eGFR) using the Japanese Eq. (22) was calculated to be $54.0 \mathrm{ml} / \mathrm{min} / 1.73 \mathrm{~m}^{2}$. At the same checkup, she was also found to have a slight hearing disturbance. In addition, she had a headache once a week since 30 years of age, and she had been diagnosed by a neurologist with migraine, which could not be controlled by triptan. Her health checkup at 32 years of age again showed proteinuria (1+) and an increased $\mathrm{sCr}(1.06 \mathrm{mg} / \mathrm{dL})$. At 33 years of age, she was referred to the Yamanashi Prefectural Central Hospital by her primary doctor in order to examine the reason for her proteinuria and decreased eGFR. At the first visit, proteinuria $(1.09 \mathrm{~g} / \mathrm{gCr})$, elevated $\mathrm{sCr}(1.28 \mathrm{mg} / \mathrm{dL})$, and elevated uric acid $(7.5 \mathrm{mg} / \mathrm{dL})$ were detected. Therefore, febuxostat and sodium bicarbonate were prescribed for the treatment of hyperuricemia. Because proteinuria and decreased renal function continued to be detected, she was admitted for a kidney biopsy to investigate the reason for proteinuria and renal dysfunction 6 months after the first visit.

On admission, her blood pressure was $114 / 70 \mathrm{mmHg}$. Physical examination showed a height of $152 \mathrm{~cm}$, body weight of $43.8 \mathrm{~kg}$, and body mass index of 19.0. No crackles or murmurs were detected on chest auscultation. There were no abnormal neurological findings. Nether skin lesions and pitting edema were detected.

The laboratory data on admission are summarized in Table 1. The remarkable data were a decreased eGFR and proteinuria. The long $\times$ short axis of the kidneys measured $101 \times 38 \mathrm{~mm}$ on the left and $104 \times 50 \mathrm{~mm}$ on the right, indicating no renal atrophy in this patient. An electrocardiogram was normal, and echocardiography showed normal cardiac function. The standard pure tone hearing test showed a right-ear value of $38.8 \mathrm{~dB}$ and a left-ear value of $40.0 \mathrm{~dB}$. She was thus diagnosed with sensorineural hearing loss by an otolaryngologist. 
Table 1

Laboratory data on admission

\begin{tabular}{|c|c|c|c|c|c|c|c|c|c|c|c|}
\hline \multicolumn{3}{|c|}{ Blood cell count } & \multicolumn{3}{|c|}{ Blood chemistry } & \multicolumn{3}{|c|}{ Immunology } & \multicolumn{3}{|c|}{ Urinalysis } \\
\hline WBC & 5400 & $/ \mu \mathrm{L}$ & TP & 6.8 & $\mathrm{~g} / \mathrm{dL}$ & $\mathrm{CH} 50$ & 54 & $\mathrm{U} / \mathrm{mL}$ & Gravity & 1.01 & \\
\hline $\mathrm{RBC}$ & 373 & $\begin{array}{l}\times 10^{4} / \\
\mu \mathrm{L}\end{array}$ & Alb & 3.9 & $\mathrm{~g} / \mathrm{dL}$ & C3 & 76.1 & $\mathrm{mg} / \mathrm{dL}$ & $\mathrm{pH}$ & 7.0 & \\
\hline $\mathrm{Hb}$ & 11.5 & $\mathrm{~g} / \mathrm{dL}$ & AST & 21 & $I U / L$ & $\mathrm{C} 4$ & 19.3 & $\mathrm{mg} / \mathrm{dL}$ & $\mathrm{RBC}$ & $1-4$ & /HPF \\
\hline $\mathrm{Ht}$ & 35.1 & $\%$ & ALT & 11 & $\mathrm{IU} / \mathrm{L}$ & $\lg G$ & 1340.3 & $\mathrm{mg} / \mathrm{dL}$ & WBC & $<1$ & /HPF \\
\hline MCV & 94.1 & $\mathrm{fl}$ & $\mathrm{LDH}$ & 186 & $\mathrm{IU} / \mathrm{L}$ & $\lg A$ & 225.7 & $\mathrm{mg} / \mathrm{dL}$ & Protein & 2.04 & $\mathrm{~g} / \mathrm{gCr}$ \\
\hline $\mathrm{MCHC}$ & 32.8 & $\%$ & ALP & 149 & $\mathrm{IU} / \mathrm{L}$ & $\lg M$ & 180.1 & $\mathrm{mg} / \mathrm{dL}$ & NAG & 8.6 & $\mathrm{U} / \mathrm{L}$ \\
\hline \multirow[t]{2}{*}{ Plt } & 25.7 & $\begin{array}{l}\times 10^{4} / \\
\mu \mathrm{L}\end{array}$ & Tbil & 0.56 & $\mathrm{mg} / \mathrm{dL}$ & ANA & $(-)$ & & & & \\
\hline & & & BUN & 21.8 & $\mathrm{mg} / \mathrm{dL}$ & $\begin{array}{l}\mathrm{M} \\
\text { protein }\end{array}$ & $(-)$ & & & & \\
\hline \multicolumn{3}{|c|}{ Coagulation } & $\mathrm{Cr}$ & 1.26 & $\mathrm{mg} / \mathrm{dL}$ & $\begin{array}{l}\mathrm{HBs} \\
\mathrm{Ag}\end{array}$ & $(-)$ & & & & \\
\hline APTT & 34.0 & $\mathrm{sec}$ & eGFR & 40.8 & $\mathrm{~mL} / \mathrm{min} / 1.73 \mathrm{~m}^{2}$ & $\begin{array}{l}\mathrm{HCV} \\
\mathrm{Ab}\end{array}$ & $(-)$ & & & & \\
\hline PT & 99.0 & $\%$ & UA & 5.4 & $\mathrm{mg} / \mathrm{dL}$ & & & & & & \\
\hline INR & 0.97 & INR & Tcho & 255 & $\mathrm{mg} / \mathrm{dL}$ & & & & & & \\
\hline \multirow[t]{3}{*}{ Fib } & 373.0 & $\mathrm{mg} / \mathrm{dL}$ & CRP & 0.01 & $\mathrm{mg} / \mathrm{dL}$ & & & & & & \\
\hline & & & FBS & 85 & $\mathrm{mg} / \mathrm{dL}$ & & & & & & \\
\hline & & & $\mathrm{HbA1c}$ & 5.3 & $\%$ & & & & & & \\
\hline \multicolumn{12}{|c|}{ Aberrant values are underlined. } \\
\hline \multicolumn{12}{|c|}{$\begin{array}{l}\text { WBC, white blood cells; RBC, red blood cells; Hb, hemoglobin; Hct, hematocrit; MCV, mean corpuscular volume; MCHC, } \\
\text { mean corpuscular hemoglobin concentration; Plt, platelet; APTT, activated partial thromboplastin time; PT, prothrombin } \\
\text { time; INR, international normalized ratio; Fib, fibrinogen, TP, total protein; Alb, albumin; AST, aspartate aminotransferase; } \\
\text { ALT, alanine aminotransferase; LDH, lactate dehydrogenase; ALP, alkaline phosphatase; Tbil, total bilirubin; BUN, blood } \\
\text { urea nitrogen; Cr, creatinine; eGFR, estimated glomerular filtration rate (by the Japanese equation); UA, uric acid; Tcho, } \\
\text { total cholesterol; CRP, C-reactive protein; FBS, fasting blood sugar; HbA1c, hemoglobin A1c (NGSP); CH50, 50\% hemolytic } \\
\text { complement activity; C3, complement 3; IgG, immunoglobulin G; ANA, antinuclear antibody; M protein, monoclonal protein; } \\
\text { HBs Ag, hepatitis B surface antigen; HCV Ab, hepatitis C virus antibody; NAG, N-acetyl glucosaminidase; HPF, high power } \\
\text { field. }\end{array}$} \\
\hline
\end{tabular}

Her parents had passed away owing to cancer (mother, breast cancer; father, colon cancer) without kidney disease or any hearing difficulty. Neither of her two older brothers had any abnormalities in their kidney function, urine, or hearing. She had a seven-year-old daughter and a five-year-old son. Her daughter's birth weight had been $2840 \mathrm{~g}$ at 41 weeks, 2 days of gestation, and her son's birth weight had been $2632 \mathrm{~g}$ at 39 weeks of gestation. Her daughter had been born by suction delivery, whereas her son had been born by normal delivery. Neither of her children had any urinary abnormalities or hearing difficulties at their medical checkups. However, the children also sometimes complained of headaches that had not yet been diagnosed by any doctor.

A percutaneous kidney biopsy under ultrasound guidance was performed. Light microscopy photographs of the renal biopsy specimens are shown in Fig. 1. Nine glomeruli were observed in the specimens. One glomerulus was globally sclerosed. Furthermore, segmental scleroses were observed at the perihilar area in two glomeruli, which was compatible with the 
perihilar variant in the Columbia classification of FSGS $(23,24)$. Interestingly, red-colored podocytes (ReCPos), whose cytoplasm was dyed red, were observed on AZAN staining. Interstitial fibrosis and tubular atrophy were observed in almost $30 \%$ of the area of the interstitium. Characteristically, granular swollen epithelial cells (GSECs), which have been reported as a specific finding of mitochondrial diseases $(25,26)$, were markedly observed in the distal tubular cells by AZAN staining (Fig. 1C). Such GSECs were emphasized by staining with anti-cytochrome c oxidase subunit 4 (COX IV) antibody (Mouse AntiCOX IV antibody [20E8C12]) (ab14744; Abcam PLC., Cambridge, UK) (Fig. 1D). COX IV staining is recognized as a loading control for mitochondria $(26,27)$. Furthermore, the sizes of the vascular smooth muscle cells in arterioles were irregular, and their arrangement was disorganized in a manner similar to that seen in older patients, despite the present patient being only 33 years old without any medical history that might cause arteriosclerosis (Fig. 1E). Such age-inappropriately disarranged and irregularly sized vascular smooth muscle cells (AiDIVs) were also noticeable in the interlobular arteries (Fig. 1F).

An immunofluorescent analysis revealed IgM and C1q deposits in the mesangial area (Fig. 1). In addition, an electron microscopic analysis (Fig. 2A-C) showed podocytes with increased mitochondria that had lost their normal cristae structure. We also detected cells with an increased content of such abnormal mitochondria among the parietal epithelial cells of the Bowman's capsule (Fig. 2D). These renal pathology findings strongly suggested FSGS due to mitochondrial disease.

When the serum lactate and pyruvate concentrations were slightly elevated at $21.0 \mathrm{mg} / \mathrm{dL}$ (normal: $3.0-17.0$ ) and $1.34 \mathrm{mg} / \mathrm{dL}$ (normal: 0.30-0.94), respectively. In addition, the sensorineural hearing difficulty and headache were also suggestive of mitochondrial disease. Therefore, we tried to perform a closer examination for mitochondrial disease.

After obtaining informed consent from the patient according to the protocol with permission from our ethics committee, genomic DNA extracted from her peripheral mononuclear blood cells and urine sediment cells was analyzed by targeted resequencing coupled with next-generation sequencing (NGS), followed by Sanger sequencing. Genomic DNA from the patient was subjected to the fragmentation and library preparation using the Lotus DNA Library Prep Kit (\#10001074; Integrated Device Technology, Inc., San Jose, CA, USA) according to the manufacturer's instructions. Target enrichment was performed with xGen Human mtDNA Research Panel (\#1075705; Integrated Device Technology) targeting the whole mtDNA and custom xGen ${ }^{\circledR}$ Predesigned Gene Capture Pools (Integrated Device Technology)/xGen Lockdown Probe pool (Integrated Device Technology) targeting the exons of 367 nuclear-encoded genes that cause mitochondrial diseases. The library was then sequenced on the Illumina MiSeq platform with MiSeq Reagent Kit v2 (MS-102-2002; Illumina, Inc., San Diego, CA, USA). As a result, a pathogenic mtDNA variant of $\mathrm{m} .13513 \mathrm{G}>\mathrm{A}$ was detected in both the patient's blood cells and urine sediment cells (Fig. 3). The Minor Variant Finder (MVF) software program (Thermo Fisher Scientific, Waltham, MA, USA) revealed that the heteroplasmy rates of the blood cells and urine sediments were 10.3\% (forward: $11.6 \% /$ reverse: $9.0 \%$ ) and $62.2 \%$ (forward: $63.7 \% /$ reverse: $60.7 \%$ ), respectively. Therefore, we diagnosed her renal dysfunction and proteinuria as being due to mitochondrial nephropathy caused by an MT-ND5 mutation.

\section{Discussion And Conclusions}

After the first case report of MELAS due to $\mathrm{m} .13513 \mathrm{G}>\mathrm{A}$ (28), reports over 40 reports have described that this variant causes various mitochondrial diseases. The variant $\mathrm{m} .13513 \mathrm{G}>\mathrm{A}$ has already been confirmed as a pathogenic variant of the mitochondrially encoded NADH dehydrogenase 5 ND5 (MT-ND5) gene by MITOMAP, a human mitochondrial genome database (https://www.mitomap.org/MITOMAP). The enzyme NADH dehydrogenase (ubiquinone) is known as Complex I, which belongs to the MRC complex. Therefore, MT-ND5 gene mutations cause mitochondrial OXPHOS disorder, which results in the occurrence of mitochondrial diseases, such as MELAS (28-30), Leigh's syndrome (29-32), and Leber's hereditary optic neuropathy (30). However, mitochondrial nephropathy due to MT-ND5 mutations was not been reported until 2020.

In 2020, the first report of three cases of mitochondrial nephropathy due to MT-ND5 mutation was published (21). In this report, the renal pathology of the three cases with the $\mathrm{m} .13513 \mathrm{G}>\mathrm{A}$ or m.13514A $>\mathrm{G}$ variant in the MT-ND5 gene mainly showed tubulo-interstitial kidney disease. Although Bakis et al. (21) assumed that one of three cases was accompanied with "secondary" FSGS, neither apparent glomerular lesions nor podocytes with abnormal mitochondria were presented in the 
report. In contrast, our present case showed apparent FSGS lesions, and we detected increased abnormal mitochondria in the glomerular visceral epithelial cells (podocytes) and glomerular parietal epithelial cells of the Bowman's capsule using electron microscopy. Therefore, with this report, it should be confirmed that MT-ND5 gene mutation can cause FSGS. Indeed, the pathogenesis of the perihilar variant of FSGS has been considered to be intraglomerular hypertension caused by hypertension or obesity, by adaptation to nephron loss owing to progression of glomerulosclerosis, or by a low nephron number owing to low birth weight (24). The present patient did not have hypertension, obesity, or any history of low birth weight. Furthermore, the diameters of all glomeruli observed under light microscopy in this patient were $<250 \mu \mathrm{m}$ (mean \pm SD; $149 \pm-72 \mu \mathrm{m}$ ). Hotta et al. (33) similarly found that the glomeruli in cases of FSGS owing to mitochondrial nephropathy were smaller than those in cases of FSGS owing to non-mitochondrial nephropathy. Therefore, we deemed the etiology of FSGS in this case to be a disorder of the OXPHOS system due to an MT-ND5 mutation and not due to "secondary" intraglomerular hypertension or glomerular hyperfiltration.

In general, mitochondrial diseases induced by mtDNA mutations vary markedly based on the differences in the rate of heteroplasmy by cell type or organ $(34,35)$. Therefore, while the reason for the differences between the present case and the previous cases with the $\mathrm{m} .13513 \mathrm{G}>\mathrm{A}$ variant could not be completely ascertained, the rate of heteroplasmy with $\mathrm{m} .13513 \mathrm{G}>$ A in podocytes might have been higher in our case than in the previous cases. Mitochondria are rich in glomerular podocytes and play important roles in maintaining podocyte function (36). In addition, it is well-known that podocyte damage results in glomerulosclerosis $(37,38)$. Therefore, we can assume that gene mutations related to MRC complexes also cause FSGS (39, 40), as energy insufficiency in podocytes can lead to podocyte damage (36).

We were unable to determine the reason for the mesangial deposits of $\operatorname{lgM}$ and $\mathrm{C} 1 \mathrm{q}$. Because $\operatorname{lgM}$ and $\mathrm{C} 1 \mathrm{q}$ are easily trapped because of their macromolecular sizes, these deposits are not expected to be immune complexes that cause inflammation or cell damage. The accumulation of more similar cases will be needed in order to discuss whether IgM and C1q deposits in the mesangium are specific for FSGS due to an MT-ND5 mutation.

It was suspected that mitochondrial nephropathy might be latent among FSGS or glomerulosclerosis cases. Therefore, we must reconsider the possibility of mitochondrial nephropathy in such cases. In the present case, the findings of abnormal mitochondria in podocytes under electron microscopy were the first clue suggesting mitochondrial nephropathy. However, in practical situations, the kidney samples for electron microscopy are small and sometimes do not include glomeruli. How are we expected to find clues suggesting mitochondrial nephropathy using these limited kidney biopsy specimens, when mitochondrial abnormalities cannot be detected by electron microscopy? In our case, GSECs with red-colored cytoplasm were also observed with AZAN staining. Because mitochondria are dyed red by acidic dyes such as AZAN, red-colored GSECs indicate that they abnormally include many mitochondria (25). Therefore, we emphasize the need to check for GSECs in cases of FSGS or glomerulosclerosis, especially in those with an unknown etiology. In addition, as COX IV is a subunit of Complex IV, COX IV staining can be used to stain mitochondria (27) and may help confirm the presence of abnormally increased numbers of mitochondria, as we previously reported $(26,41)$. Furthermore, AiDIVs were observed in the arterioles and intralobular arteries in this case. Such findings have also been reported in other cases of mitochondrial nephropathy (13, 26). Interestingly, ReCPos were detected by AZAN staining as we recently reported them in a case of mitochondrial nephropathy (27). Similar to GSECs, ReCPos are considered to abnormally include many mitochondria in their cytoplasm. In summary, in cases of FSGS or glomerulosclerosis with an unknown etiology, the existence of GSECs, AiDIVs, and ReCPos should be carefully evaluated, and COX IV staining may aid in the detection of abnormally increased mitochondrial counts.

This is the first case report of mitochondrial nephropathy with apparent glomerular lesions caused by an MT-ND5 gene mutation. Furthermore, this is the first known case of FSGS with the gene mutation encoding subunit proteins of Complex I. In addition, we propose that not only GSECs but also AiDIVs and ReCPos should be clues to find out mitochondrial nephropathies, which could be latent in etiology-unknown FSGS or glomerulosclerosis cases.

\section{List Of Abbreviations}


NADH dehydrogenase 5, ND5; mitochondrially encoded ND5, MT-ND5; oxidative phosphorylation, OXPHOS; mitochondrial encephalomyopathy, lactic acidosis, and stroke-like episodes, MELAS; focal segmental glomerulosclerosis, FSGS; granular swollen epithelial cells, GSECs; age-inappropriately disarranged and irregularly sized vascular smooth muscle cells, AiDIVs; red-colored podocytes, ReCPos; adenosine triphosphate, ATP; mitochondrial respiratory chain, MRC; mitochondrial respiratory chain disorder, MRCD; mitochondrial DNA, mtDNA; nuclear DNA, nDNA; creatinine, Cr; serum creatinine, sCr; estimated glomerular filtration rate, eGFR; cytochrome c oxidase subunit 4, COX IV

\section{Declarations}

\section{Acknowledgements}

We thank MinakoTominaga and Eri Gomi for providing technical assistance.

\section{Ethics Declarations}

\section{Ethics approval and consent to participate}

This study was approved by the Ethics Committee of the Chiba Children's Hospital (2014-11-05). The patient provided informed consent and gave permission to participate in this study.

\section{Consent for publication}

All authors consented to the publication of the manuscript in Diagnostic Pathology.

\section{Competing interests}

The authors declare that they have no competing interests.

\section{Funding}

This work was supported in part by the Practical Research Project for Rare/Intractable Diseases from the Japan Agency for Medical Research and Development, AMED (19ek0109273, 20ek0109468) (http://www.amed.go.jp/en/) to TI, YO, and KM.

\section{Author Contributions}

Drafting of manuscript, TN; coordination of study and draft preparation, Tl; clinical information, IN and MW; pathological analysis, $\mathrm{KH}$; molecular genetic studies, $\mathrm{YY}$ and $\mathrm{YK}$; sequence alignment and data curation, $\mathrm{YO}$; coordination of study and data curation, KM; coordination of study and clinical information, YJ. The authors have read and approved the final manuscript.

\section{Availability of data and materials}

Most of the data generated or analyzed during this study are included in this published article.

\section{References}

1. Skladal D, Halliday J, Thorburn DR. Minimum birth prevalence of mitochondrial respiratory chain disorders in children. Brain. 2003;126:1905-1912.

2. Ohtake A, Murayama K, Mori M, Harashima H, Yamazaki T, Tamaru S, et al. Diagnosis and molecular basis of mitochondrial respiratory chain disorders: exome sequencing for disease gene identification. Biochim Biophys Acta. 2014;1840(4):1355-1359.

3. Dimauro S, Davidzon G. Mitochondrial DNA and disease. Ann. Med. 2005;37:222-232. 
4. Kirby DM, Thorburn DR. Approaches to finding the molecular basis of mitochondrial oxidative phosphorylation disorders. Twin Res. Hum. Genet. 2008;11(4):395-411.

5. Leigh D. Subacute necrotizing encephalomyelopathy in an infant. J Neurol Neurosurg Psychiatry. 1951;14(3):216-221.

6. Mimaki M, Wang X, McKenzie M, Thorburn DR, Ryan MT. Understanding mitochondrial complex I assembly in health and disease. Biochim Biophys Acta. 2012;1817(6):851-862.

7. Yatsuga S, Povalko N, Nishioka J, Katayama K, Kakimoto N, Matsuishi T, et al. MELAS: a nationwide prospective cohort study of 96 patients in Japan. Biochim Biophys Acta. 2012;1820(5):619-624.

8. Bates MG, Bourke JP, Giordano C, d'Amati G, Turnbull DM, Taylor RW. Cardiac involvement in mitochondrial DNA disease: clinical spectrum, diagnosis, and management. Eur Heart J. 2012;33(24):3023-3033.

9. Lee WS, Sokol RJ. Mitochondrial hepatopathies: advances in genetics and pathogenesis. Hepatology. 2007;45(6):15551565.

10. Olmos PR, Borzone GR, Olmos JP, Diez A, Santos JL, Serrano V, et al. Mitochondrial diabetes and deafness: possible dysfunction of strial marginal cells of the inner ear. J Otolaryngol Head Neck Surg. 2011;40(2):93-103.

11. Suzuki S. Diabetes mellitus with mitochondrial gene mutations in Japan. Ann N Y Acad Sci. 2004;1011:185-192.

12. Thorner PS, Balfe JW, Becker LE, Baumal R. Abnormal mitochondria on a renal biopsy from a case of mitochondrial myopathy. Pediatr Pathol. 1985;4(1-2):25-35.

13. Mochizuki H, Joh K, Kawame H, Imadachi A, Nozaki H, Ohashi T, et al. Mitochondrial encephalomyopathies preceded by de-Toni-Debré-Fanconi syndrome or focal segmental glomerulosclerosis. Clin Nephrol. 1996;46(5):347-352.

14. Quinzii C, Naini A, Salviati L, Trevisson E, Navas P, DiMauro S, et al. A mutation in para-hydroxybenzoate-polyprenyl transferase (COQ2) causes primary coenzyme Q10 deficiency. Am J Hum Genet. 2006;78:345-349.

15. Diomedi-Camassei F, Di Giandomenico S, Santorelli FM, Caridi G, Piemonte F, Montini G, et al. COQ2 nephropathy: a newly described inherited mitochondriopathy with primary renal involvement. J Am Soc Nephrol. 2007;18(10):27732780 .

16. López LC, Schuelke M, Quinzii CM, Kanki T, Rodenburg RJ, Naini A, et al. Leigh syndrome with nephropathy and CoQ10 deficiency due to decaprenyl diphosphate synthase subunit 2 (PDSS2) mutations. Am J Hum Genet. 2006;79(6):11251129.

17. Heeringa SF, Chernin G, Chaki M, Zhou W, Sloan AJ, Ji Z, et al. COQ6 mutations in human patients produce nephrotic syndrome with sensorineural deafness. J Clin Invest. 2011;121(5):2013-2024.

18. Ashraf S, Gee HY, Woerner S, Xie LX, Vega-Warner V, Lovric S, et al. ADCK4 mutations promote steroid-resistant nephrotic syndrome through CoQ10 biosynthesis disruption. J Clin Invest. 2013;123(12):5179-5189.

19. Lemoine S, Panaye M, Rabeyrin M, Errazuriz-Cerda E, Mousson de Camaret B, Petiot $P$, et al. Renal involvement in neuropathy, ataxia, retinitis pigmentosa (NARP) syndrome: A case report. Am J Kidney Dis. 2018;71(5):754-757.

20. Fervenza FC, Gavrilova RH, Nasr SH, Irazabal MV, Nath KA. CKD due to a novel mitochondrial DNA mutation: A case report. Am J Kidney Dis. 2019;73(2):273-277.

21. Bakis H, Trimouille A, Vermorel A, Redonnet I, Goizet C, Boulestreau R, et al. Adult onset tubulo-interstitial nephropathy in MT-ND5-related phenotypes. Clin Genet. 2020;97(4):628-633.

22. Matsuo S, Imai E, Horio M, Yasuda Y, Tomita K, Nitta K, et al. Revised equations for estimated GFR from serum creatinine levels in Japan. Am J Kidney Dis. 2009;53:982-992.

23. D'Agati VD, Fogo AB, Bruijn JA, Jennette JC. Pathologic classification of focal segmental glomerulosclerosis: a working proposal. Am J Kidney Dis. 2004;43(2):368-82.

24. Tsuchimoto A, Matsukuma Y, Ueki K, Tanaka S, Masutani K, Nakagawa K, et al. Utility of Columbia classification in focal segmental glomerulosclerosis: renal prognosis and treatment response among the pathological variants. Nephrol Dial Transplant 2019 Jan 11. doi: 10.1093/ndt/gfy374. [Epub ahead of print] 
25. Kobayashi A, Goto Y, Nagata M, Yamaguchi Y. Granular swollen epithelial cells: a histologic and diagnostic marker for mitochondrial nephropathy. Am J Surg Pathol. 2010;34(2):262-270.

26. Imasawa T, Tanaka M, Yamaguchi Y, Nakazato T, Kitamura H, Nishimura M. 7501 T > A mitochondrial DNA variant in a patient with glomerulosclerosis. Ren Fail. 2014;36(9):1461-1465.

27. Mahad DJ, Ziabreva I, Campbell G, Lax N, White K, Hanson PS, et al. Mitochondrial changes within the axons in multiple sclerosis. Brain. 2009;132(Pt 5):1161-1174.

28. Santorelli FM, Tanji K, Kulikova R, Shanske S, Vilarinho L, Hays AP, et al. Identification of a novel mutation in the mtDNA ND5 gene associated with MELAS. Biochem Biophys Res Commun. 1997;238(2):326-328.

29. Pulkes T, Eunson L, Patterson V, Siddiqui A, Wood NW, Nelson IP, et al. The mitochondrial DNA G13513A transition in ND5 is associated with a LHON/MELAS overlap syndrome and may be a frequent cause of MELAS. Ann Neurol. 1999;46(6):916-919.

30. Chol M, Lebon S, Bénit P, Chretien D, de Lonlay P, Goldenberg A, et al. The mitochondrial DNA G13513A MELAS mutation in the NADH dehydrogenase 5 gene is a frequent cause of Leigh-like syndrome with isolated complex I deficiency. J Med Genet. 2003;40(3):188-91.

31. Carelli V, La Morgia C, Valentino ML, Barboni P, Ross-Cisneros FN, Sadun AA. Retinal ganglion cell neurodegeneration in mitochondrial inherited disorders Biochim Biophys Acta. 2009;1787(5):518-28.

32. Ogawa E, Fushimi T, Ogawa-Tominaga M, Shimura M, Tajika M, Ichimoto K, et al. Mortality of Japanese patients with Leigh syndrome: Effects of age at onset and genetic diagnosis. J Inherit Metab Dis. 2020 Jan 22. doi:

10.1002/jimd.12218. [Epub ahead of print]

33. Hotta O, Inoue CN, Miyabayashi S, Furuta T, Takeuchi A, Taguma Y. Clinical and pathologic features of focal segmental glomerulosclerosis with mitochondrial tRNALeu(UUR) gene mutation. Kidney Int. 2001;59(4):1236-1243.

34. van den Ameele J, Li AYZ, Ma H, Chinnery PF. Mitochondrial heteroplasmy beyond the oocyte bottleneck. Semin Cell Dev Biol. 2020;97:156-166.

35. Geng X, Zhang Y, Yan J, Chu C, Gao F, Jiang Z, et al. Mitochondrial DNA mutation m.3243A>G is associated with altered mitochondrial function in peripheral blood mononuclear cells, with heteroplasmy levels and with clinical phenotypes. Diabet Med. 2019;36(6):776-783.

36. Imasawa T, Rossignol R. Podocyte energy metabolism and glomerular diseases. Int J Biochem Cell Biol. 2013;45(9):2109-2118.

37. Kriz W, Gretz N, Lemley KV. Progression of glomerular diseases: is the podocyte the culprit? Kidney Int. 1998;54(3):687697.

38. Nagata M. Podocyte injury and its consequences Kidney Int. 2016;89(6):1221-1230.

39. O'Toole JF. Renal manifestations of genetic mitochondrial disease. Int J Nephrol Renovasc Dis. 2014;7:57-67.

40. Lim K, Steele D, Fenves A, Thadhani R, Heher E, Karaa A. Focal segmental glomerulosclerosis associated with mitochondrial disease. Clin Nephrol Case Stud. 2017;5:20-25.

41. Imasawa T, Tanaka M, Maruyama N, Kawaguchi T, Yamaguchi Y, Rossignol R, et al. Pathological similarities between low birth weight-related nephropathy and nephropathy associated with mitochondrial cytopathy. Diagn Pathol. 2014;9:181.

42. Maeoka Y, Doi T, Aizawa M, Miyasako K, Hirashio S, Masuda Y, et al. A case report of adult-onset COQ8B nephropathy presenting focal segmental glomerulosclerosis with granular swollen podocytes. BMC Nephrol. 2020;21(1):376.

\section{Figures}



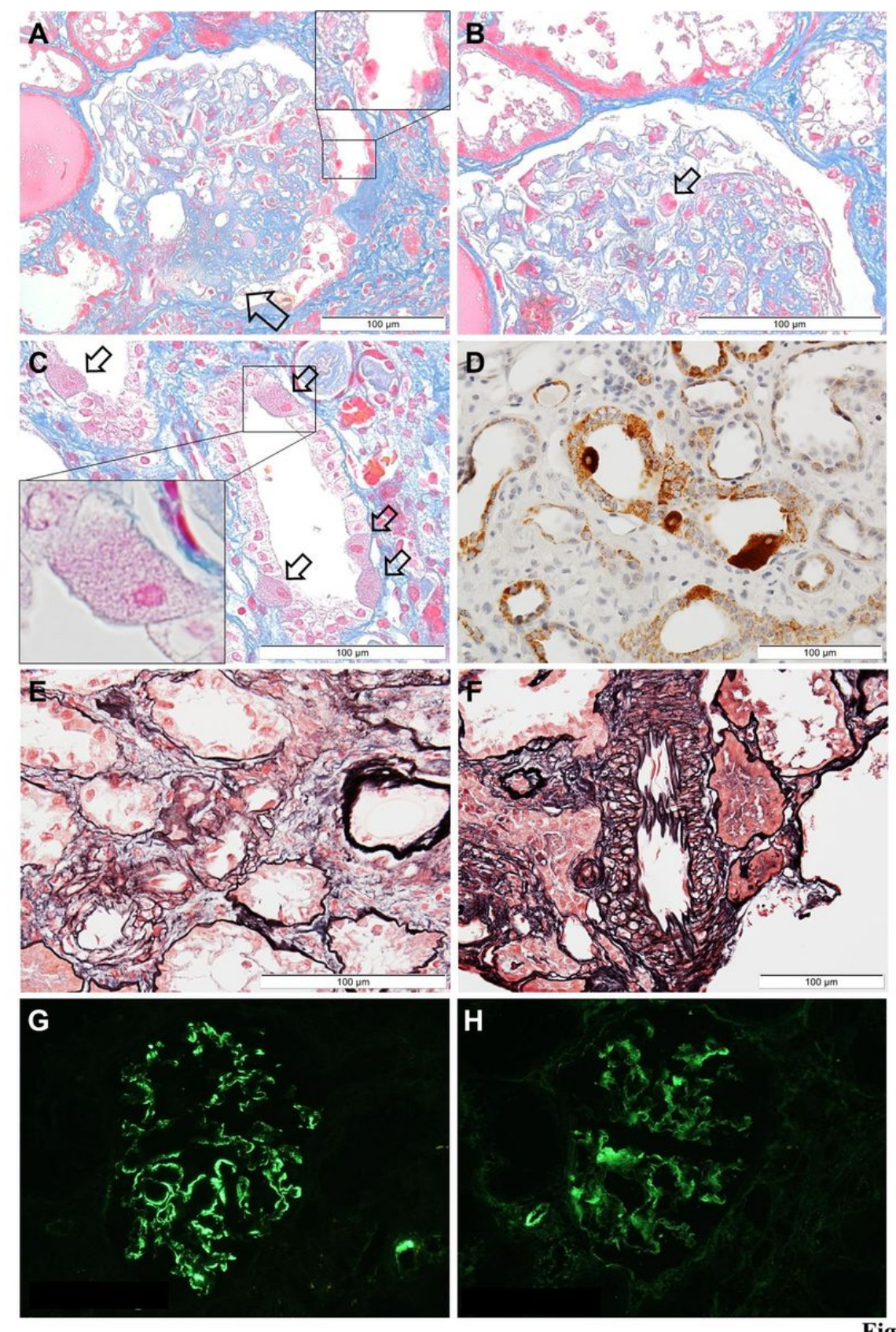

Figure 1

\section{Figure 1}

Light microscopy and immunofluorescent findings (A): The segmental sclerosis lesion at the perihilar area is indicated by an arrow. A red-colored podocyte (ReCPo) is surrounded by a square, which is magnified at the top right (Azan stain). (B): The ReCPo is indicated by an arrow (AZAN stain). (C): GSECs in the distal tubuli are indicted by arrows (AZAN stain). One GSEC is magnified at the lower left. (D): Staining of COX IV emphasizes tubular cells, including many mitochondria, which correspond to GSECs. (E): In the afferent arteriole that connects to the glomerulus of (A), the sizes of the vascular smooth muscle cells are irregular, and their arrangement is disorganized, similar to the appearance seen in older patients (PAM-HE stain). (F): Ageinappropriately disarranged and irregularly sized vascular smooth muscle cells (AiDIVs) are observed in an interlobular artery (PAM-HE stain). (G): IgM deposits are detected in the mesangium. (H): C1q deposits are detected in the mesangium. 

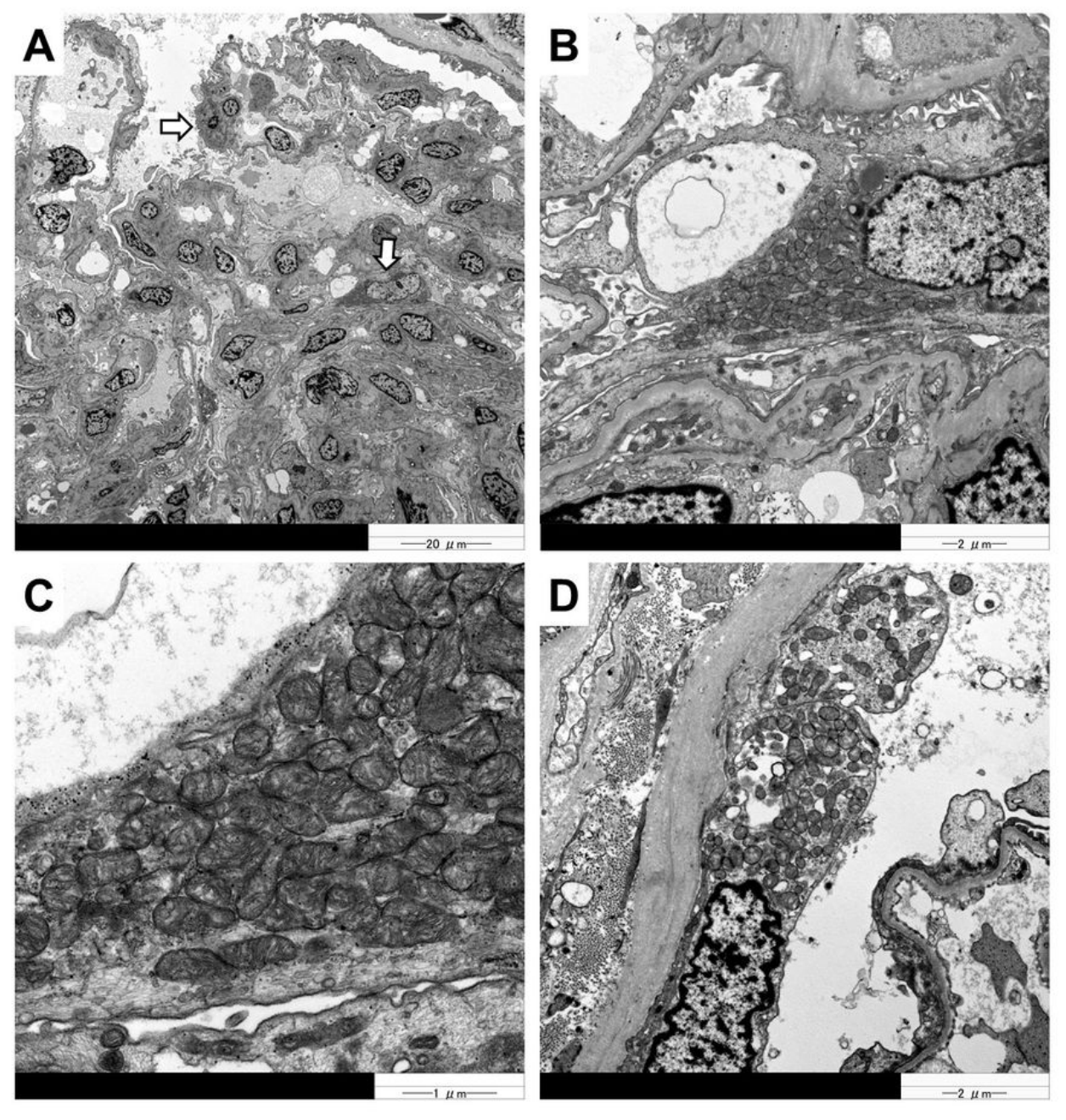

Figure 2

\section{Figure 2}

Electron microscopy findings (A): Arrows indicate podocytes filled with increased mitochondria. (B): In a podocyte, abnormally high numbers of mitochondria are seen in the cytoplasm. (C): Magnification of (B) reveals that the increased mitochondria have lost their organized cristae structure. (D): The parietal epithelial cells are also filled with mitochondria. 
a

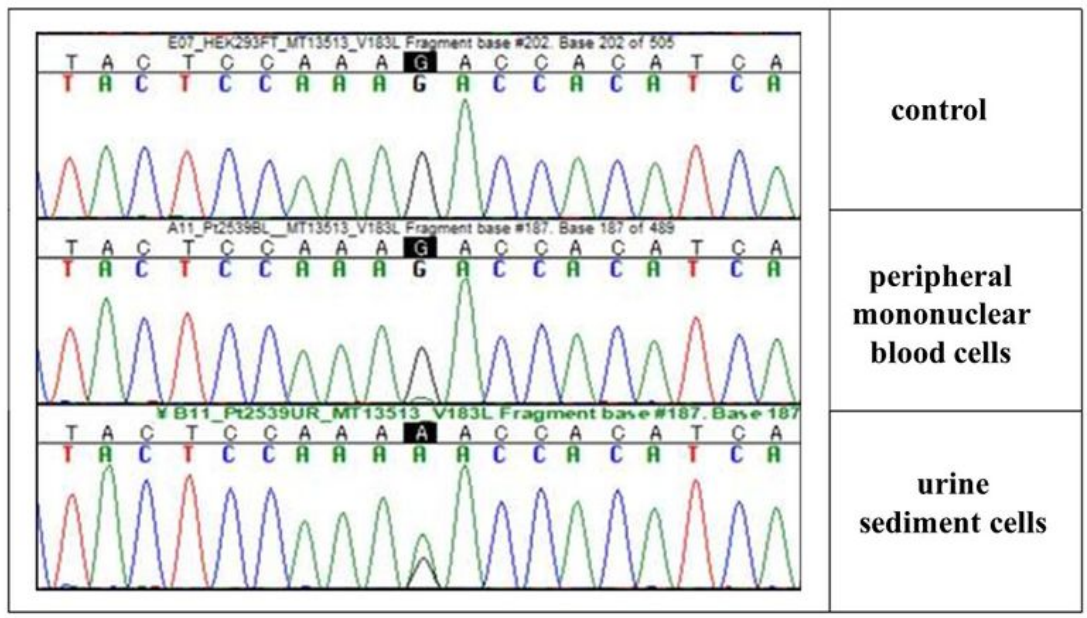

b
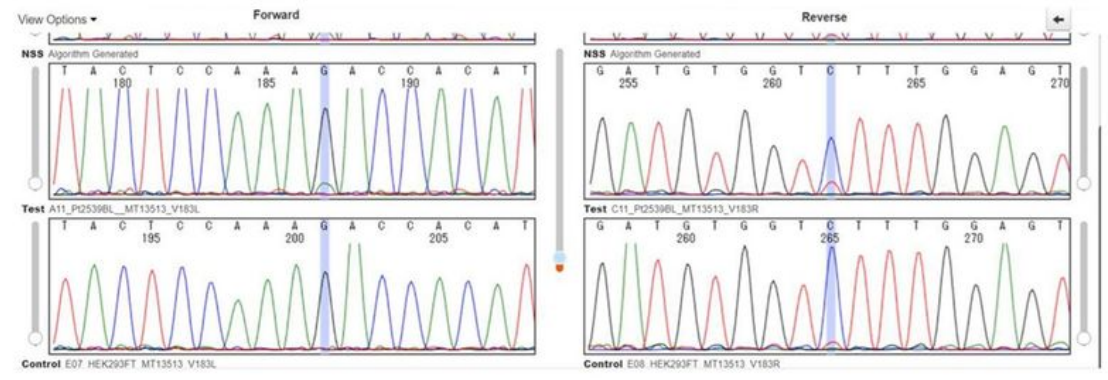

C
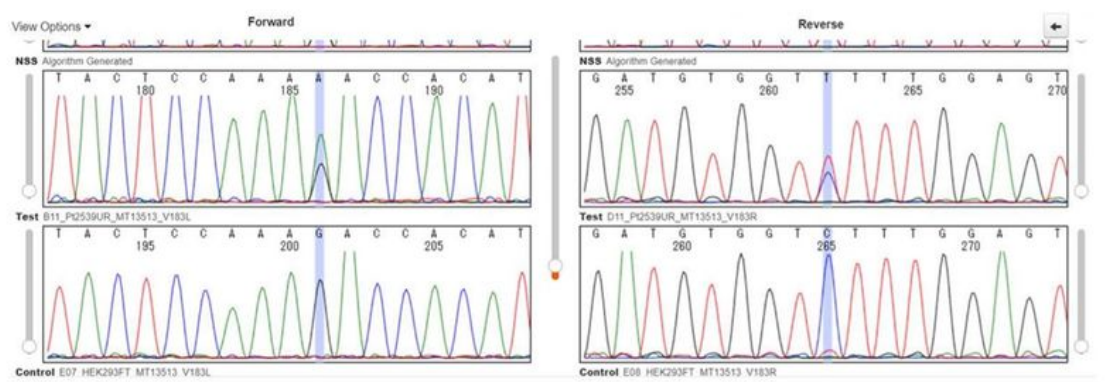

Figure 3

Figure 3

Results of a genetic analysis (A): The electropherogram from Sanger sequencing indicates that the patient has the $\mathrm{m} .13513$ G>A variant in her peripheral mononuclear blood cells and urine sediment cells. (B): The rate of heteroplasmy calculated by the MVF software program is $10.3 \%$ (forward: $11.6 \% /$ reverse: $9.0 \%$ ) in peripheral mononuclear blood cells (upper panel:

patient, lower panel: control). (C): The rate of heteroplasmy is $62.2 \%$ (forward: $63.7 \% /$ reverse: $60.7 \%$ ) in urine sediment cells (upper panel: patient, lower panel: control).

\section{Supplementary Files}

This is a list of supplementary files associated with this preprint. Click to download.

- checklistofCAREguidelinesImasawa.pdf 